\title{
Solvent-salt synergy offers a safe pathway towards next generation high voltage Li-ion batteries
}

\author{
Guozhong Cao
}

Driven by expanding applications in mobile electronics, grid storage, and electric vehicles, the global lithium-ion battery market is projected to reach $>45$ billion dollars by 2022 [1]. While each of these applications has a distinct set of energy storage requirements (lifetime, safety, cost, volume, weight), a significant market share would directly benefit from the availability of high voltage $(>4.5 \mathrm{~V})$ lithium ion batteries (LIBs) with enhanced power and energy density. Cathode chemistries of $5 \mathrm{~V}$ class which offer these improved theoretical metrics are readily available, as exemplified by the $4.6 \mathrm{~V}$ lithium nickelmanganese oxide (LNMO) and $4.8 \mathrm{~V}$ lithium cobalt phosphate (LCP); however, their commercial application is limited by the lack of a compatible electrolyte. When cycled with the hallmark carbonate-based electrolytes that have been used as workhorse by LIB industry since its birth, LNMO/graphite cells exhibit significant gassing and rapid capacity fade as a result of the electrolyte's thermodynamic instability above $\sim 4.5 \mathrm{~V} v s$. lithium metal. High energy electrode couples and large pack formats also intensify safety requirements, demanding robust battery management systems (BMS) or other safety mechanisms unless an intrinsically safe, non-flammable electrolyte is developed and used. Together, these considerations underscore that a safe, high voltage compatible electrolyte is a critical prerequisite for lithium-ion batteries to reach full maturity as an energy storage technology capable of supporting current and future market demand.

In order to address this need, a bold departure from the status quo for lithium-ion battery electrolytes is necessary and imperative. Sulfones generally offer a promising alternative to carbonate solvents due to their intrinsic oxidative stability, comparably high dielectric constants, and high flashpoint. Generally, previous work on sulfones for LIBs can be fit into three categories: (A) fundamental studies [2-4], (B) sulfone-based electrolytes tested with
Li-Ni-Mn-Co oxides (NMC)/graphite cells [5-7], and (C) sulfone-based electrolytes tested with LNMO/lithium titanate (LTO) cells [8]. The general results from the fundamental studies are that the oxidative stability of sulfones is structurally invariant but can be augmented with salt concentration, and that sulfones are not independently adequate solid electrolyte interphase (SEI)formers, which is an important distinction from carbonate-based solvents. The published literature in category $B$ addresses the sulfone-graphite incompatibility with the addition of SEI-forming additives such as vinylene carbonate (VC) or fluoroethylene carbonate (FEC), but within a limited voltage window due to the limited oxidative stability of these additives. In category $\mathrm{C}$, the electrolytes are optimized for stability with high voltage cathodes such as LNMO, but coupled with LTO (a high voltage anode) to avoid the SEI issue of these electrolytes. Interestingly, up to this point, researchers have been unable to successfully couple LNMO/graphite with a sulfone-based electrolyte.

That changed recently with a new report from Alvarado, Schroeder and their coworkers [9], who presented an elegantly simple yet electrochemically robust, two component electrolyte formulation consisting of lithium bis(fluorosulfonyl)imide (LiFSI) salt in sulfolane (SL). At the anode, early-onset reduction of the salt anion results in formation of a LiF-rich interphase, which effectively suppresses solvent co-intercalation and subsequent graphite exfoliation, enabling unprecedented and highly reversible graphite cycling in a pure sulfone system. Under oxidative conditions, quantum chemistry (QC) calculations predict complex/aggregate formation as a result of increasing salt concentration which slows the decomposition of SL and leads to polymerization rather than gaseous products-a fundamentally significant improvement over carbonate solvents.

Department of Materials Science and Engineering, University of Washington, Seattle, WA 98195-2120, USA

Email: gzcao@uw.edu 
a

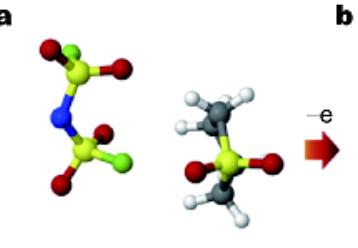

b

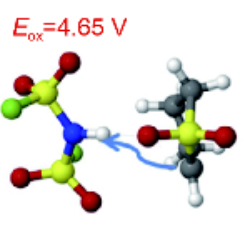

f
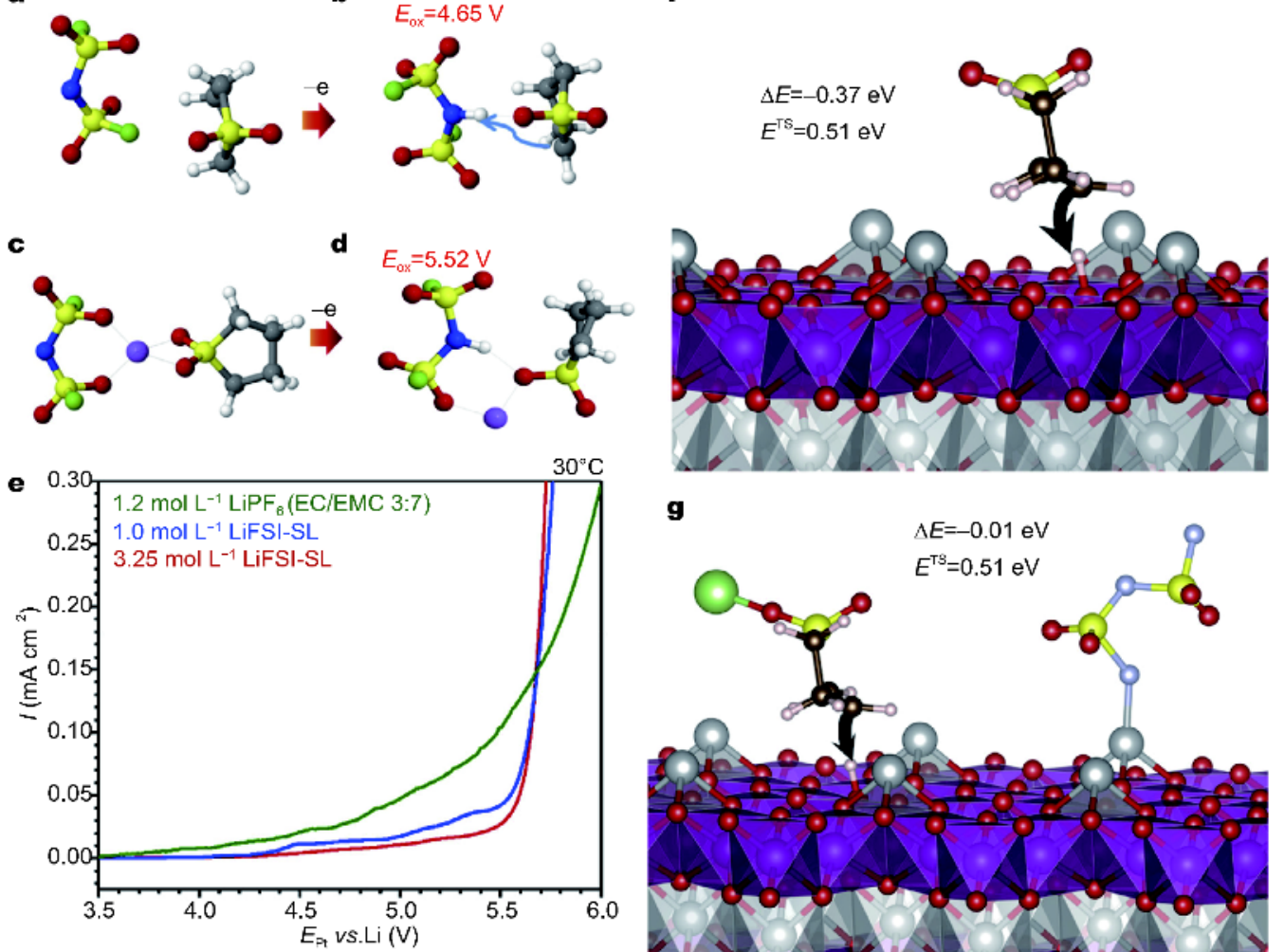

C

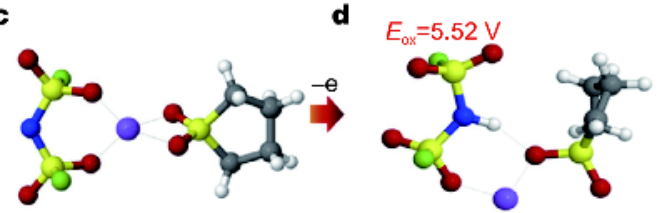

$30^{\circ} \mathrm{C}$

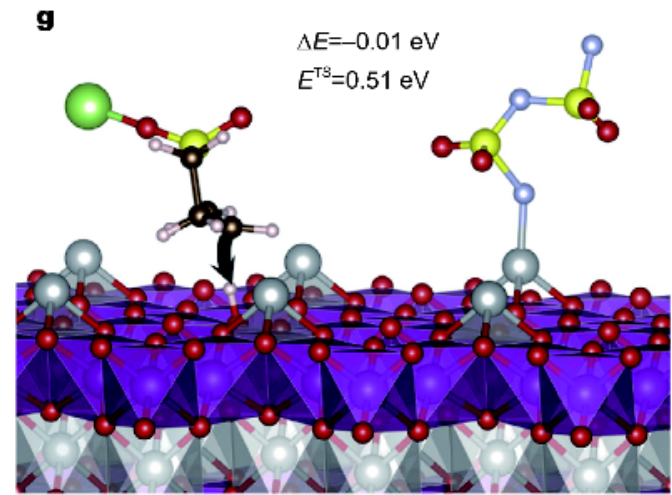

Figure 1 (a-d) Oxidation potential $\left(E_{\mathrm{ox}}\right)\left(v s . \mathrm{Li} / \mathrm{Li}^{+}\right.$) from QC calculations with clusters surrounded by SMD (SL) implicit solvent model, (e) Htransfer reaction from SL, and (f, g) Li-SL separated from FSI to the $\mathrm{Ni}_{0.5} \mathrm{Mn}_{1.5} \mathrm{O}_{4}$ cathode surface from PBE $+\mathrm{U} D F T$ calculations. $\Delta E$ and $E^{\mathrm{TS}}$ are the $\mathrm{H}$-transfer reaction energy and barrier, respectively.

Fig. 1a and $\mathrm{b}$ are the QC calculation results that suggest the oxidation stability of the SL (FSI ${ }^{-}$) complexes, surrounded by implicit solvent with SL parameters, to be $4.65 \mathrm{~V} v s$. $\mathrm{Li} / \mathrm{Li}^{+}$as a result of $\mathrm{H}$-transfer during oxidation from SL to nitrogen of FSI. This oxidation process is attributed to the initial small peak observed in the LSV around $4.5 \mathrm{~V}$ (Fig. 1e). The important role of the $\mathrm{H}$ transfer during oxidation by cathode surfaces is known for the carbonates and ether/FSI complexes, but not in the SL-based electrolytes. In the concentrated regime when all SL molecules are coordinated by $\mathrm{Li}^{+}$, QC calculations predict that the oxidation potential for the LiFSI-SL complexes significantly increases from 4.6 to $5.5 \mathrm{~V}$ (Fig. 1c, d). Fig. If and g show the SL and $\mathrm{Li}^{+}-\mathrm{SL}$ interactions with the low energy (111) surface of fully charged $\mathrm{Ni}_{0.5} \mathrm{Mn}_{1.5} \mathrm{O}_{4}$ cathode.

The researchers corroborate these predictions with solid experimental data and evidences using a variety of advanced characterization methods. X-ray photoelectron spectroscopy (XPS), cryogenic scanning transmission electron microscopy (cryo-(S)TEM), and electron energy loss spectroscopy (EELS) results revealed the presence of a thin, conformal, sulfur-based cathode electrolyte interphase (CEI). Together, the functional interphases (SEI/ CEI) generated by this electrolyte system supported long term operation of an aggressive high voltage $(4.85 \mathrm{~V})$ LNMO/graphite full cell, which retained $\sim 70 \%$ of its original first-cycle discharge capacity after the $1,000^{\text {th }}$ cycle. While further optimization of this electrolyte is necessary for commercial applications (i.e., viscosity, wetting, low temperature performance, $\mathrm{Al}$ corrosion), the promising preliminary results of this two component system provide a strong starting point for continued development of safe high voltage electrolytes.

Received 4 May 2018; accepted 5 May 2018;

published online 23 May 2018

1 Singh R. Lithium-Ion Battery Market by Material Type (Cathode, Anode, Electrolytic solution, Foils, Binders, Separators) and Industry Vertical (Smartphones, UPS, Cars, Aircraft, Busses, Trucks, Mining equipment, Smart Grid, Games, Gardening tools)—Global Opportunity Analysis and Industry Forecast. 2015-2022 EN 
161380, 2016

2 Borodin $\mathrm{O}$, Behl W, Jow TR. Oxidative stability and initial decomposition reactions of carbonate, sulfone, and alkyl phosphatebased electrolytes. J Phys Chem C, 2013, 117: 8661-8682

3 Wang Y, Xing L, Li W, et al. Why do sulfone-based electrolytes show stability at high voltages? Insight from density functional theory. J Phys Chem Lett, 2013, 4: 3992-3999

$4 \mathrm{Xu} \mathrm{K}$, Angell CA. Sulfone-based electrolytes for lithium-ion batteries. J Electrochem Soc, 2002, 149: A920

5 Xia J, Self J, Ma L, et al. Sulfolane-based electrolyte for high voltage $\mathrm{Li}\left(\mathrm{Ni}_{0.42} \mathrm{Mn}_{0.42} \mathrm{Co}_{0.16}\right) \mathrm{O}_{2}$ (NMC442)/graphite pouch cells. J Electrochem Soc, 2015, 162: A1424-A1431
6 Hilbig P, Ibing L, Wagner R, et al. Ethyl methyl sulfone-based electrolytes for lithium ion battery applications. Energies, 2017, 10: 1312

7 Su CC, He M, Redfern PC, et al. Oxidatively stable fluorinated sulfone electrolytes for high voltage high energy lithium-ion batteries. Energy Environ Sci, 2017, 10: 900-904

8 Abouimrane A, Belharouak I, Amine K. Sulfone-based electrolytes for high-voltage Li-ion batteries. Electrochem Commun, 2009, 11: 1073-1076

9 Alvarado J, Schroeder MA, Zhang M, et al. A carbonate-free, sulfone-based electrolyte for high-voltage Li-ion batteries. Mater Today, 2018, 21: 341-353 